\title{
Binucleation of Drosophila Adult Male Accessory Gland Cells Increases Plasticity of Organ Size for Effective Reproduction
}

\author{
Kiichiro Taniguchi' ${ }^{1}$, Akihiko Kokuryo ${ }^{2,3}$, Takao Imano $^{2,3}$, Ryunosuke Minami ${ }^{4}$, Hideki Nakagoshi ${ }^{4}$ and Takashi Adachi-Yamada ${ }^{1,2,3, *}$
}

1Department of Life Science, Faculty of Science, Gakushuin University, Tokyo 171-8588, Japan

2Institute for Biomolecular Science, Gakushuin University, Tokyo 171-8588, Japan

3Department of Biology, Graduate School of Science, Kobe University, Kobe 657-8501, Japan

4Graduate School of Natural Science and Technology, Okayama University, Okayama 700-8530, Japan

\begin{abstract}
Although most animal cells possess only a single nucleus, some cells such as myocytes and hepatocytes show sporadic occurrences of multiple nuclei. The Drosophila adult male accessory gland, an internal reproductive organ for production of seminal fluid components, displays an exceptional columnar epithelium where all cells have two nuclei. Despite this striking feature, no adaptive significances to account for binucleation have been proposed.

We demonstrate that one possible purpose of binucleation is enabling plasticity in organ size. To compare various cytological traits between binucleate and mononucleate states, we artificially converted the cellular state from the binucleate state to either the endoreplicated mononucleate or pseudodivided mononucleate state in the accessory gland epithelium by genetically manipulating the spindle assembly checkpoint. Depending on the flies' age and mating and feeding conditions, the apical area of the binucleate cells enlarges compared with that of endoreplicated mononucleate cells. On the other hand, the apical area shrinks after mating compared with that of divided mononucleate cells. Consequently, this wide range of apical area size in binucleate cells increases the plasticity in organ size compared with that in endoreplicated mononucleate and divided mononucleate cells. The highly plastic features in an organ composed of binucleate cells should lead to higher rates of reproductive success in response to nutrition uptake and mating frequency. Similar plasticity might also be beneficial in hepatocytes and myocytes, both of which show great variability in cell size. Our results provide direct evidence that cell-size plasticity is a basis of multinucleation in animal cells.
\end{abstract}

Keywords: Drosophila; Male accessory gland; Binucleation; Multinucleation; Organ size; Cell shape; Reproduction

Abbreviations: ACPs: Accessory Gland Proteins; ISC: Intestinal Stem Cell; EB: Enteroblast; EC: Enterocyte; SAC: Spindle Assembly Checkpoint; AE: After Eclosion; Cora: Coracle; Ac-H3: Acetylated Histone $\mathrm{H} 3$

\section{Introduction}

Although most cells that compose animal organs contain single nuclei, certain kinds of cells are multinucleated. Multinucleation is known to be caused by either cell fusion (as in skeletal muscle or placental trophoblast) or mitosis without cytokinesis (as in hepatocytes or myocytes) and is thought to be a process of tissue/cell growth. In this case, the numbers of chromosome copies and nuclei in each cell increase. In other cases of tissue/cell growth such as cell division and endoreplication (chromosomal doubling without karyokinesis), only a single nucleus per cell exists. It is known that binucleation of hepatocytes or myocytes occurs according to the growth of individuals. In rat hepatocytes, the proportion of binucleate cells increase in the transition from suckling to weaning, and the rate of binucleation is controlled by nutrient signaling [1]. In rat myocytes, cells predominantly binucleate by 10 days after birth [2]. However, the functional benefits for organs by increasing the number of nuclei are largely unclear.

To address this question, we used the Drosophila male accessory gland, which is composed of binucleate cells, as a model for studying bunucleation (Figure 1A and 1B). The male accessory gland increases reproductive success; it produces more than 80 peptide species (accessory gland proteins (ACPs)) and sends them together with sperm into the female reproductive organs [3]. The ACPs stored in female spermatheca alter various female reproductive phenotypes such as frequency of oviposition and rejection of further mating with other males [3]. The accessory gland lobe is composed of paved and predominant (ca. 1000) main cells and scattered (ca. 40) secondary cells [4]. Both cell types are thought to play distinct but as yet unknown roles, and they are both binucleate [4] (Figure 1B).

Here, we show the benefit of the binucleate state for reproductive success in the male accessory gland. We found that binucleation enables the cells to have a wider range of flexibility in size and shape by changing the position of the two nuclei. This cellular plasticity provided great variability in the lumenal size of the organ, depending on nutritional and mating states.

\section{Materials and Methods}

\section{Drosophila strains}

Canton-S and $w^{1118}$ were used as wild-type strains. chico $^{f p 147 E}$, a deletion mutant lacking the coding region of chico [5] was used. The following Gal4 expressing lines were used: esg ${ }^{\text {NP6267 }}$ (esg-Gal4, Gal4 Enhancer Trap Insertion Database (GETDB), http://kyotofly.kit. jp/stocks/GETDB/getdb.html), which expresses Gal4 in the adult secondary cells, and $f n g^{N P 5399}$ (fng-Gal4, GETDB), which expresses

*Corresponding author: Takashi Adachi-Yamada, Department of Life Science, Faculty of Science, Gakushuin University, Tokyo 171-8588, Japan, Tel: +81-3-39860221; Fax: +81-3-5992-1029; E-mail: Takashi.Adachi-Yamada@gakushuin.ac.jp

Received December 01, 2011; Accepted December 06, 2011; Published December 08, 2011

Citation: Taniguchi K, Kokuryo A, Imano T, Minami R, Nakagoshi $\mathrm{H}$, et al. (2012) Binucleation of Drosophila Adult Male Accessory Gland Cells Increases Plasticity of Organ Size for Effective Reproduction. Biol Syst Open Access 1:e101. doi:10.4172/2329-6577.1000e101

Copyright: (c) 2012 Taniguchi K, et al. This is an open-access article distributed under the terms of the Creative Commons Attribution License, which permits unrestricted use, distribution, and reproduction in any medium, provided the original author and source are credited. 
Gal4 in the entire pupal accessory gland epithelial cells. AyGal4 was described previously [6]. $h s-F L P$ was used as the source of the FLP recombinase [7]. UAS-InR.IR (UAS-InR.RNAi) and UAS-mad2.IR (UAS-mad2.RNAi), express inverted repeat RNAs, which form hairpin loop double strand RNA for InR and mad2, respectively. Histone H2Avariant::RFP [8], were described previously. The following Drosophila species were obtained from Kyorin University (Japan) and were used in the evolutionary analysis: $D$. simulans, $D$. erecta, D. ananassae, $D$. pseudoobscura, D. persimilis, D. willistoni, D. virillis and D. grimshawi. All fly stocks except for D. grimshawi were maintained on a standard fly medium at $25^{\circ} \mathrm{C}$. The samples of $D$. grimshawi were dead carcasses stored in ethanol.

\section{Immunostaining and microscopic analysis}

The adult dissected accessory glands were fixed under a severe condition (4\% formaldehyde (Wako) /PBS for 30 minutes) and stained

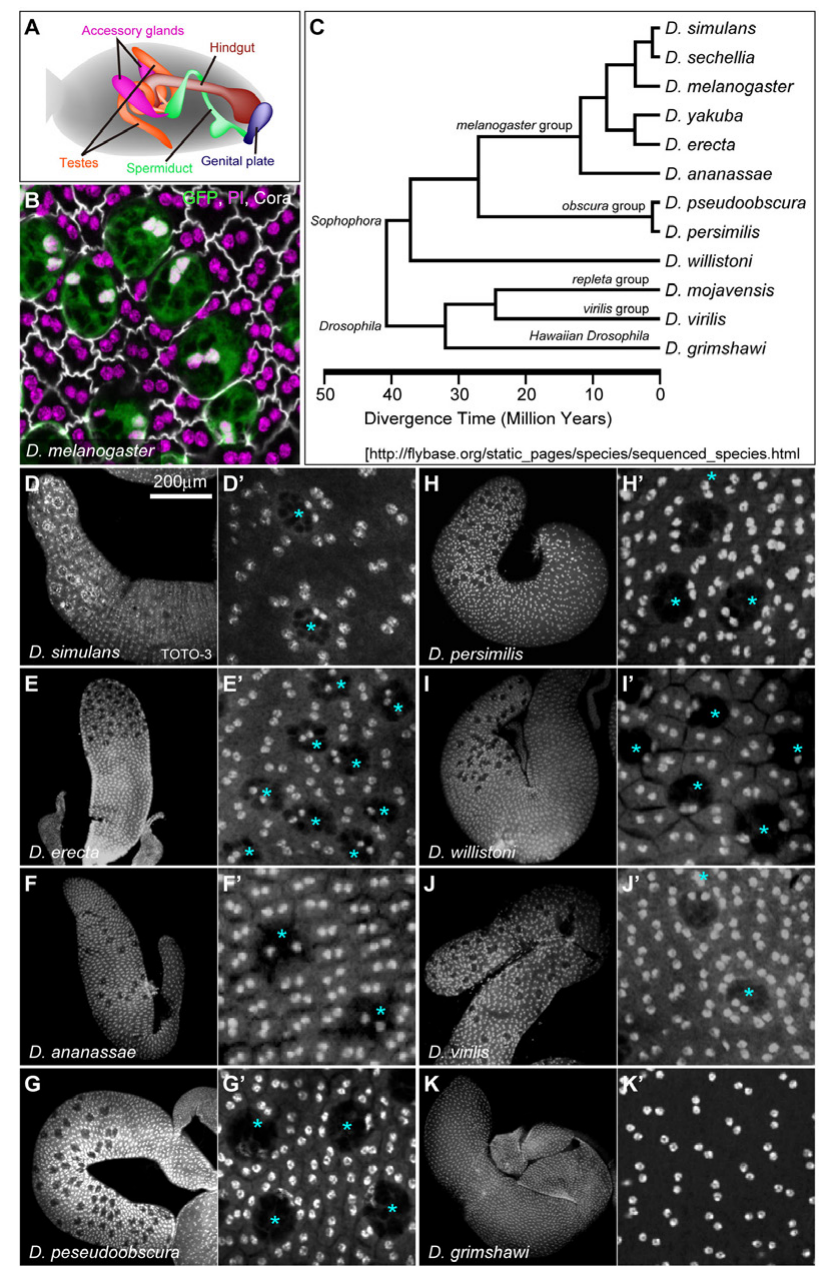

Figure 1: Binucleation of male accessory gland epithelial cells in fruit fly species. (A) An illustration of adult male abdomen showing the reproductive systems around the hindgut. Posterior is to the right. (B) Adult accessory gland epithelium labeled with PI (magenta), GFP (green) driven by esg-gal4 (secondary cells), and anti-Cora antibody (blue). (C) Phylogenetic tree of the genome-sequenced 12 species of Drosophila. (D-K and D'-K') Whole (D-K) and magnified ( $D^{\prime}-K^{\prime}$ ) images of accessory glands labeled with TOTO-3 (white) in representative species: $D$. simulans $\left(\mathrm{D}, \mathrm{D}^{\prime}\right), \mathrm{D}$. erecta $\left(\mathrm{E}, \mathrm{E}^{\prime}\right), D$. ananassae $(\mathrm{F}$, $\left.\mathrm{F}^{\prime}\right)$, D. pseudoobscura $\left(\mathrm{G}, \mathrm{G}^{\prime}\right), \mathrm{D}$. persimilis $\left(\mathrm{H}, \mathrm{H}^{\prime}\right), \mathrm{D}$. willistoni $\left(\mathrm{I}, \mathrm{I}^{\prime}\right), \mathrm{D}$. virillis $\left(\mathrm{J}, \mathrm{J}^{\prime}\right)$ and $D$. grimshawi $\left(\mathrm{K}, \mathrm{K}^{\prime}\right)$. Cyan asterisks in ( $\left.\mathrm{D}^{\prime}-\mathrm{J}^{\prime}\right)$ indicate secondary cells which are absent in $D$. grimshawi. with standard immunostaining protocols. For the DNA staining, the fixed samples were pretreated with RNase (Wako, $0.025 \mathrm{mg} / \mathrm{ml}$ ) for 15 minutes at $37^{\circ} \mathrm{C}$. Propidium iodide (Invitrogen, 1:500) or TOTO-3 iodide (Invitrogen, 1:1000) were used for DNA staining. The following primary antibodies were used: Guinea Pig anti-Paired polyclonal antibody (Asian Distribution Center for Segmentation Antibodies [9], 1:200), Rabbit anti-acetyl-Histone H3 (Ac-H3) polyclonal antibody (Millipore, 1:200), Mouse anti-Coracle (Cora) monoclonal antibody (Developmental Studies Hybridoma Bank, 1:20). The following secondary antibodies were used: Cy3-conjugated Donkey anti-Mouse IgG (Jackson ImmunoResearch, 1:200), Cy2-conjugated Donkey anti-Mouse IgG (Jackson ImmunoResearch, 1:200), Cy5-conjugated Donkey anti-Mouse IgG (Jackson ImmunoResearch, 1:200), Cy3conjugated Donkey anti-Rabbit IgG. Stained samples were mounted in 50\% glycerol/PBS containing $0.25 \% \mathrm{n}$-propyl gallate (Wako) and observed with an ECLIPSE TE2000-U with a Digital ECLIPSE C1 and $\mathrm{C} 1 \mathrm{Si}$ confocal system (Nikon). Images were processed using EZ-C1 Gold Version 3.70 (Nikon), Adobe Photoshop CS3 Extended (Adobe Systems) and Adobe Illustrator CS3 (Adobe Systems).

\section{Measurement of fluorescent Intensity of Histone H2A- Variant::RFP}

To analyze the intensity of RFP, the color images were converted into gray-scale images, and the "Integrated Density" data from Image J 1.41o (National Institute of Health, http://rsb.info.nih.gov/ij/) were used to measure the fluorescent intensities. The sums of the values obtained from the z-section image series were taken to be "fluorescent intensities" of the DNA in nuclei (Figure S1).

\section{Construction of Acp70A::mRFP lines}

The $m R F P$ fragment was excised from $p T u b 84 B-m R F P$ (gifted from K. Matsuno) and subcloned into pattB (FlyC31, http://flyc31.frontiersin-genetics.org/) vector to construct pattB-mRFP. The region from -598 to +4 of $A c p 70 A$, which includes its enhancer element and core promoter, was amplified with PCR and subcloned into pattB- $m R F P$ to construct $p A c p 70 A:: m R F P$-attB (Figure S2A). Transgenic lines carrying $p A c p 70 A:: m R F P$-attB was generated with $\varphi C 31$-mediated site-specific integration [10].

\section{Measurement of apical area sizes of cells and horizontal distances between two nuclei}

Outlines of cells and nuclei were labeled with anti-Cora antibody and Propidium iodide, respectively, and the z-section images were stacked. The areas encircled by anti-Cora antibody labeling (plasma membrane) are taken to be the apical areas of cells, and the distances between the centroids of two nuclei were taken to be the horizontal distances between the two nuclei, by using Image J 1.41o (National Institute of Health). The ratios of each datum value to the mean of all data values in $4 \mathrm{~N} \times 2$ cells were used to normalize inconsistencies in accessory gland volumes between individuals. To test statistical significance, regression analyses of both data sets were performed. Coefficient of correlations ( $r$-values), linear regressive equations, $\mathrm{P}$-values, and t-values were also calculated.

\section{Set-up for mating males with females}

Adult males of Canton-S or UAS-mad2.RNAi/+; fng-Gal4/+ at 9 days $\mathrm{AE}$ were added to a vial containing a large number of adult virgin females, and the vial was incubated at $25^{\circ} \mathrm{C}$ for 12 hours. 


\section{Results and Discussion}

\section{Binucleation of male accessory gland epithelial cells are evolutionally conserved}

We first characterized polyploidy of male accessory gland epithelial cells. We measured the fluorescent intensity of Histone $\mathrm{H} 2 \mathrm{~A}$ variant::RFP in each nucleus. For normalization of intensities, the adult midgut intestinal stem cells/enteroblasts, early-enterocytes, and enterocytes were used as references of diploid, tetraploid, and octaploid cells, respectively (supplementary Figure S1A-C and S1F). As a result, the accessory gland epithelialium was composed of octaploid cells with two tetraploid nuclei (supplementary Figure S1D-F). Given that binucleation in accessory gland epithelial cells resulted from mitosis without cytokinesis (data not shown and manuscript in preparation), a single round of endoreplication is thought to take place after binucleation during the maturation of cells.

We next observed the male accessory gland epithelial cells of various Drosophila species from subgenus Sophophora (D. simulans, D erecta, D.ananassae, D. pseudoobscura, D. persimilis, D. willistoni) and subgenus Drosophila (D. virillis, D. grimshawi) (Figure 1C) to determine the evolutionary conservation of binucleation. We predicted that binucleation in the male accessory gland is strongly conserved during evolution if binucleation is adopted for certain benefits. As a result, binucleation was observed in all species we observed (Figure $1 \mathrm{D}-\mathrm{K}$ and $1 \mathrm{D}^{\prime}-\mathrm{K}$ '). It is also known that binucleation in the male accessory gland also occurs in the mid-fly [11] and Queensland fruit fly [12]. These results suggest that binucleation in male accessory gland epithelium is an evolutionally conserved state in a wide variety of fruit flies and is expected to have certain functional benefits for this organ.

Number of nuclei is not involved in the functional differentiation of male accessory gland epithelial cells

What is the physiological significance of binucleation? Does binucleation bring more benefits to cells than endoreplication or cell division do? Do binucleate cells show highly produce ACPs than these mononucleate cells? To address these questions, we compared various cytological traits between cells with different nuclear phenotypes. In the accessory gland cells of a hypomorphic polo mutant flies where various nuclear phenotypes were produced (Figure 2) by defects in chromatid segregation [13], we did not find any differences in the gene expression characteristics between normal binucleate and abnormal nuclear phenotype cells (no differences in histone acetylation [14] in chromatin modification (Figure 2B-D compared with 2A), expression of transcription factor Paired [15] which is important for accessory gland function (Figure 2F-H compared with $2 \mathrm{E}$ ), or expression of Acp70A [16] (Figure 2J-L compared with 2I)). These results indicate that the number of nuclei did not affect proper gene expression.

\section{Positions of two nuclei correlate with conversion of cell-shape from squamous to cuboidal}

The accessory gland enlarges its volume to retain more ACPs; thereby, increasing reproductive success [17-19]. In contrast, in certain starved and/or postmating conditions, the accessory gland represses its cell growth and/or shrinks the apical area of the cells to reduce seminal fluid storage in order to save systemic energy (starved condition) and empty out its cavity (postmating condition) (supplementary Figure S3A-F). To adapt to these various situations, it would be beneficial if the accessory gland could easily change its volume. We found that the binucleate cells changed the size of their apical area, which contributed

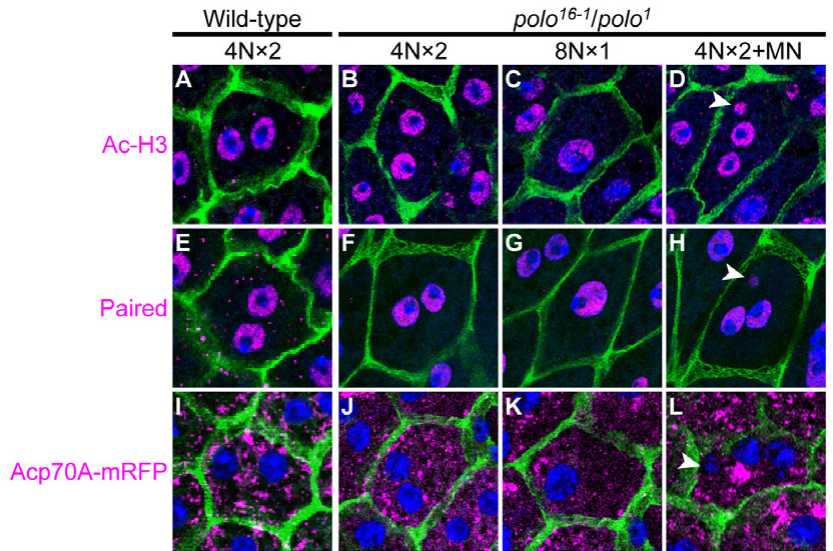

Figure 2: Binucleation is dispensable for functional differentiations of secretory cells in the accessory gland. (A-L) Main cells in adult accessory gland epithelium in wild-type $(\mathrm{A}, \mathrm{E}, \mathrm{I})$ and transheterozygote of polo ${ }^{16-1}$ and polo $^{1}$ (B-D, F-H, J-L) labeled with anti-Cora antibody (green), TOTO-3 (blue) and various differentiation markers of accessory glands (magenta) indicated at the left.
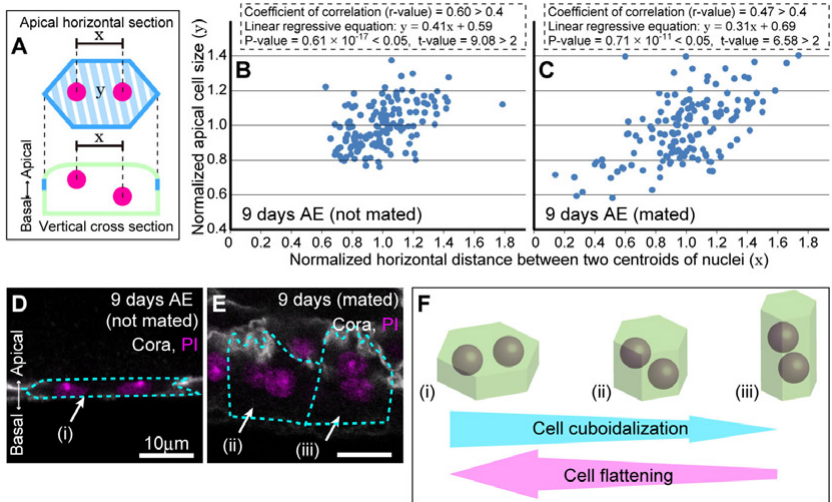

Figure 3: The positions of two nuclei correlate with the cell shape in male accessory gland epithelium. (A) Schematic diagram of a main cell in the accessory gland, showing the horizontal distance between the two centroids of the nuclei $(x)$ and cell size $(y)$. En-face view of apical-horizontal section (upper) and cross-sectional view (lower) are shown. Green line and magenta circles indicate outlines of the cell and two nuclei, respectively. Blue lines indicate the apical areas labeled with anti-Cora antibody, for measuring the cell size (y). (B and $C$ ) Scatter diagrams representing the relationship of the horizontal distance between the two nuclei $(x)$ to cell size $(y)$. Data were collected from adult accessory glands on day 9 after eclosion (AE) without having mated (B) and 12 hours after mating $(C)$. ( $D$, and $E$ ) Cross-sectional views of adult main cells on day $9 \mathrm{AE}$ without having mated $(\mathrm{D})$ and 12 hours after mating $(E)$, labeled with anti-Cora and PI. Arrows indicate three representatives of cells with the nuclei in various positional relationships: (i) an expanded cell, in which the two nuclei do not overlap, (ii) a moderately shrunken cell, in which the nuclei overlap, (iii) a heavily shrunken cell, in which one nucleus is layered on top of the other. Outlines of cells are indicated by dashed lines.

to changing the lumenal volume of the organ, through a shift in the three-dimensional positions of the two nuclei. In fact, the accessory glands and their main cells showed great variability in the apical area and three-dimensional positions of the two nuclei, depending on age and feeding conditions (Figure 3A-E and data not shown). Regression analysis also indicated a positive correlation between the change in the position of the nuclei and cell apical area (Figure 3B and 3C). After mating to exhaust seminal fluid, the accessory glands apparently shrunk as a result of nuclei overlapping and layering (Figure 3E 

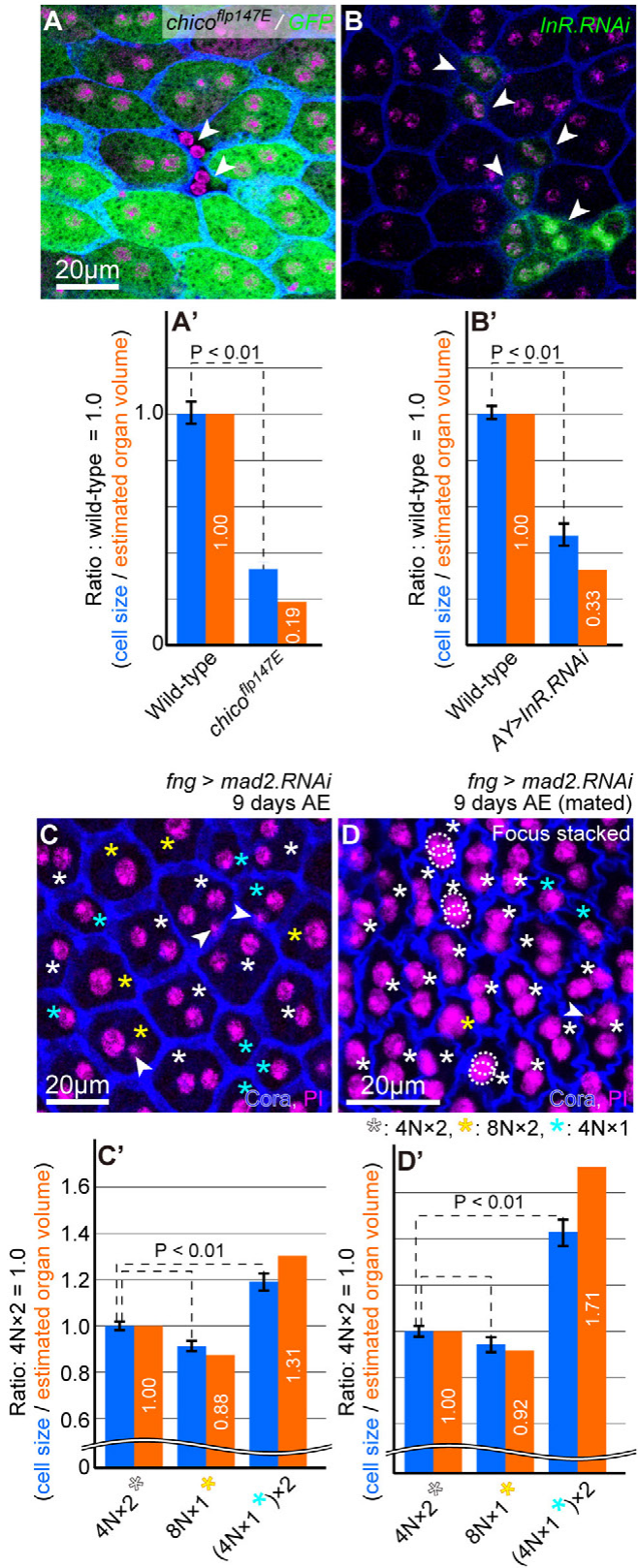

Figure 4: Binucleation enables the accessory gland to change its lumenal size more easily. ( $A$ and $B$ ) An adult main cell clone mutant for chico encoding the insulin receptor substrate homolog (lacking GFP, arrowheads) (A) and Flipout clones with RNAi-mediated depletion of InR encoding the insulin recepto homolog (expressing GFP, arrowheads) (B) labeled with PI (magenta), GFP (green), and anti-Cora antibody (blue). Scale bars: $20 \mu \mathrm{m}$. (A' and B') Bar graphs representing normalized means of apical area of the main cells (blue bars) and estimated volume of the accessory gland lumen (supplementary Figure S5) (orange bars) in the cases of chico mutant (A) and InR.RNAi (B)

( $C$ and $D$ ) Adult main cells, in which mad2 is knocked down by using the fng-Gal4 labeled with anti-Cora antibody and PI. Adult main cells on day $9 \mathrm{AE}$ without having mated $(C)$ and 12 hours after mating (D) are shown. (D) is a focusstacked image for displaying overlapping nuclei. Dashed lines indicate outlines of two overlapping nuclei. Nuclei in the circular muscle cells are indicated by arrowheads. Membrane ruffling is also apparent in the main cells after mating (D). Scale bars: $20 \mu \mathrm{m}$. (C' and D') Bar graphs represent normalized means of the apical area in various nuclear types of main cells in the accessory gland where mad2 is knocked down (blue bars). Estimated volumes of accessory gland lumen are shown each right (orange bars). Adult male flies at day $9 \mathrm{AE}$ without having mated (C') and 12 hours after mating (D') are shown. compared with 3D) and plasma membrane ruffling (Figure 3E and 4D compared with 3D and 4C). Furthermore, when nutrient signaling (e.g. insulin) was blocked [20], these cells displayed much more shrinkage compared with the cells of other organs, suggesting that the capacity of the accessory gland is tightly regulated by the nutrient state (Figure $4 \mathrm{~A}$, A', B and B'). Thus, the lumenal volume of the male accessory gland can be changed easily through conversion of cell shape from squamous to cuboidal, which is in accordance with the relocalization of two nuclei (Figure 3F).

\section{Binucleation is effective strategy for higher plasticity in accessory gland size}

Given that the cell-apical area correlated with the positions of the two nuclei, we hypothesized that the binucleate cells provide more plasticity in the apical area compared with endoreplicated or divided mononucleate cells. To examine this hypothesis, we induced both cell types by knockdown of mad2, a gene encoding a factor of the spindle assembly checkpoint (SAC), which monitors the attachment of microtubules with kinetochores to ensure equal segregation of chromatids [21]. It has been reported that Drosophila SAC does not function in standard mitosis as frequently as in mammals unless the spindle function is perturbed [22]. However, we discovered that accessory gland binucleation required the SAC function during chromosome segregation. Therefore, approximately half the population of the main cells exhibited a mononucleate state through simple knockdown of mad2 under a non-stressful condition (Figure 4C). These mononucleate cells showed two major surges in their DNA content; one was octaploid and the other tetraploid (supplementary Figure S4). In the octaploid mononucleate cells ("endoreplicated mononucleate cells"), karyokinesis (as well as cytokinesis) was assumed to be skipped at the final M-phase. In the tetraploid mononucleate cells (i.e., pseudodivided mononucleate cells), karyokineses in the final M-phase and subsequent endoreplication were assumed to be skipped.

The apical size of cells and lumenal size of the organ (supplementary Figure S5) were measured just after the main cells had fully expanded before mating on day 9 after eclosion (Figure 4C). The measurements showed that the lumenal size of the organ composed of the binucleate cells was approximately $114 \%(=100 \times 100 / 88)$ of the organ composed of endoreplicated mononucleate cells (Figure 4C and 4C'). However, pseudodivided mononucleate cells caused an even larger increase in organ size than binucleate cells (Figure $4 \mathrm{C}$ and $4 \mathrm{C}^{\prime}$ ). Nevertheless, this may not automatically mean that cell division is the best option for reproductive success because higher retention of ACPs does not always provide advantages to males in, for example, female-absent, starved, and/or postmating states. That is, the lumenal size of the organ composed of the binucleate cells was approximately $58 \%(=100$ $\mathrm{x} 100 / 171$ ) of that composed of pseudodivided mononucleate cells after mating on day 9 after eclosion. Thus, cell division does not seem to be an appropriate strategy for cell shrinkage (Figure 4D and 4D'). These results suggest that binucleation is an effective strategy for higher plasticity in the apical area of cells to increase reproductive success (Figure 5).

Based on the above findings, a wider range of plasticity in cell size seems to be a unique benefit of binucleation. Insulin-regulated binucleation was reported in mammalian liver, which dramatically changes size depending on dietary conditions $[1,23]$. The findings presented here are new insights into the significance of binucleation in view of the link between cell shape and organ size control. 
Citation: Taniguchi K, Kokuryo A, Imano T, Minami R, Nakagoshi H, et al. (2012) Binucleation of Drosophila Adult Male Accessory Gland Cells Increases Plasticity of Organ Size for Effective Reproduction. Biol Syst Open Access 1:e101. doi:10.4172/2329-6577.1000e101

Page 5 of 5

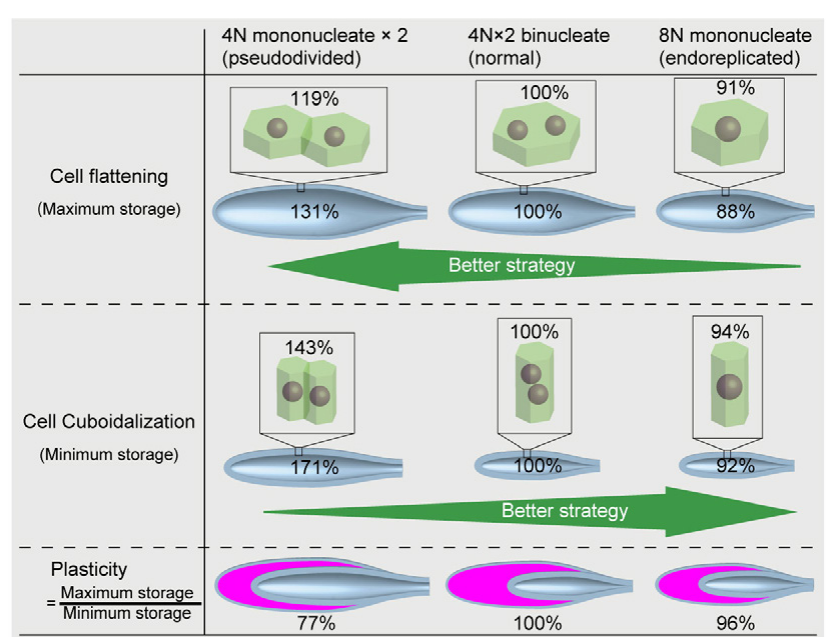

Figure 5: Binucleation increases the plasticity of the cell shape, leading to a flexible lumenal size of accessory gland. Pseudodivided mononucleate cells (double $4 \mathrm{~N}$ ) can lead to more expanded gland in response to aging and feeding although it is not good at shrinking. On the other hand, endoreplicated mononucleate $(8 \mathrm{~N})$ cells can lead to more shrunken gland in response to starvation although it is not good at expanding. Percentages in the organ lumens represent the calculated sizes of the accessory glands relative to wild type (binucleate) one which is normalized to 100 . Percentages under the magenta lumens represent the plasticities in the accessory gland size relative to wild type (binucleate) one which is normalized to 100 .

\section{Acknowledgements}

We thank T. Aigaki, the Bloomington Stock Center, Kyoto Stock Center, KYORIN-fly, the National Institute of Genetics, and the VDRC Stock Center for the fly stocks; Y. Hiromi, the Asian Distribution Center for Segmentation Antibodies, and Developmental Studies of Hybridoma Bank for the antibodies. We also thank BestGene Inc. for generating new transgenic fly lines. We are grateful to M. F. Wolfner and J. Sitnik for their helpful discussion.

\section{References}

1. Celton-Morizur S, Merlen G, Couton D, Margall-Ducos G, Desdouets C (2009) The insulin/Akt pathway controls a specific cell division program that leads to generation of binucleated tetraploid liver cells in rodents. J Clin Invest 119: 1880-1887.

2. Wulfsohn D, Nyengaard JR, Tang Y (2004) Postnatal growth of cardiomyocytes in the left ventricle of the rat. Anat Rec A Discov Mol Cell Evol Biol 277: 236247.

3. Carvalho GB, Kapahi P, Anderson DJ, Benzer S (2006) Allocrine modulation of feeding behavior by the Sex Peptide of Drosophila. Curr Biol 16: 692-696.

4. Bertram MJ, Akerkar GA, Ard RL, Gonzalez C, Wolfner MF (1992) Cell typespecific gene expression in the Drosophila melanogaster male accessory gland. Mech Dev 38: 33-40.

5. Bohni R, Riesgo-Escovar J, Oldham S, Brogiolo W, Stocker H, et al. (1999) Autonomous control of cell and organ size by $\mathrm{CHICO}$, a Drosophila homolog of vertebrate IRS1-4. Cell 97: 865-875

6. Ito K, Awano W, Suzuki K, Hiromi Y, Yamamoto D (1997) The Drosophila mushroom body is a quadruple structure of clonal units each of which contains a virtually identical set of neurones and glial cells. Development 124: 761-771.

7. Golic KG, Lindquist S (1989) The FLP recombinase of yeast catalyzes sitespecific recombination in the Drosophila genome. Cell 59: 499-509.

8. Heeger S, Leismann O, Schittenhelm R, Schraidt O, Heidmann S, et al. (2005) Genetic interactions of separase regulatory subunits reveal the diverged Drosophila Cenp-C homolog. Genes Dev 19: 2041-2053.

9. Kosman D, Small S, Reinitz J (1998) Rapid preparation of a panel of polyclonal antibodies to Drosophila segmentation proteins. Dev Genes Evol 208: 290-294.
10. Groth AC, Fish M, Nusse R, Calos MP (2004) Construction of transgenic Drosophila by using the site-specific integrase from phage $\varphi C 31$. Genetics 166: $1775-1782$

11. Marchini D, Del Bene G, Cappelli L, Dallai R (2003) Ultrastructure of the male reproductive accessory glands in the medfly Ceratitis capitata (Diptera: Tephritidae) and preliminary characterization of their secretions. Arthropod Struct Dev 31: 313-327.

12. Radhakrishnan P, Marchini D, Taylor PW (2009) Ultrastructure of male reproductive accessory glands and ejaculatory duct in the Queensland fruit fly, Bactrocera tryoni (Diptera: Tephritidae). Arthropod Struct Dev 38: 216-226.

13. Llamazares S, Moreira A, Tavares A, Girdham C, Spruce BA, et al. (1991) polo encodes a protein kinase homolog required for mitosis in Drosophila. Genes Dev 5: 2153-2165.

14. Lee DY, Hayes JJ, Pruss D, Wolffe AP (1993) A positive role for histone acetylation in transcription factor access to nucleosomal DNA. Cell 72: 73-84.

15. Xue L, Noll M (2002) Dual role of the Pax gene paired in accessory gland development of Drosophila. Development 129: 339-346.

16. Chen PS, Stumm-Zollinger E, Aigaki T, Balmer J, Bienz M, et al. (1988) A male accessory gland peptide that regulates reproductive behavior of female D. melanogaster. Cell 54: 291-298.

17. Wigby S, Sirot LK, Linklater JR, Buehner N, Calboli FC, et al. (2009) Semina fluid protein allocation and male reproductive success. Curr Biol 19: 751-757.

18. Bangham J, Chapman T, Partridge L (2002) Effects of body size, accessory gland and testis size on pre- and postcopulatory success in Drosophila melanogaster. Animal Behaviour 64: 915-921.

19. Crudgington HS, Fellows S, Badcock NS, Snook RR (2009) Experimenta manipulation of sexual selection promotes greater male mating capacity but does not alter sperm investment. Evolution 63: 926-938.

20. Coelho CM, Leevers SJ (2000) Do growth and cell division rates determine cell size in multicellular organisms? J Cell Sci 113: 2927-2934.

21. Musacchio A, Salmon ED (2007) The spindle-assembly checkpoint in space and time. Nat Rev Mol Cell Biol 8: 379-393.

22. Buffin E, Emre D, Karess RE (2007) Flies without a spindle checkpoint. Nat Cell Biol 9: 565-572.

23. Margall-Ducos G, Celton-Morizur S, Couton D, Bregerie O, Desdouets C (2007) Liver tetraploidization is controlled by a new process of incomplete cytokinesis. J Cell Sci 120: 3633-3639. 